University of Nebraska - Lincoln

DigitalCommons@University of Nebraska - Lincoln

Faculty Publications in Food Science and Technology

Food Science and Technology Department

January 2004

\title{
A consensus protocol for the determination of the threshold doses for allergenic foods: how much is too much?
}

\author{
S. L. Taylor \\ University of Nebraska-Lincoln, staylor2@unl.edu \\ S. L. Hefle \\ University of Nebraska-Lincoln \\ C. Bindslev-Jensen \\ Odense University Hospital, Odense, Denmark
}

F. M. Atkins

National Jewish Medical and Research Center, Denver, CO, USA

C. Andre

Stallergenes, Antony, France

See next page for additional authors

Follow this and additional works at: https://digitalcommons.unl.edu/foodsciefacpub

Part of the Food Science Commons

Taylor, S. L.; Hefle, S. L.; Bindslev-Jensen, C.; Atkins, F. M.; Andre, C.; Bruijnzeel-Koomen, C.; Burks, A. W.; Bush, R. K.; Ebisawa, M.; Eigenmann, P. A.; Host, A.; Hourihane, Jonathan O. B.; Isolauri, E.; Hill, D. J.; Knulst, A.; Lack, G.; Sampson, H. A.; Moneret-Vautrin, D. A.; Rance, F.; Vadas, P. A.; Yunginger, J. W.; Zeiger, R. S.; Salminen, J. W.; Madsen, C.; and Abbott, P., "A consensus protocol for the determination of the threshold doses for allergenic foods: how much is too much?" (2004). Faculty Publications in Food Science and Technology. 36.

https://digitalcommons.unl.edu/foodsciefacpub/36

This Article is brought to you for free and open access by the Food Science and Technology Department at DigitalCommons@University of Nebraska - Lincoln. It has been accepted for inclusion in Faculty Publications in Food Science and Technology by an authorized administrator of DigitalCommons@University of Nebraska - Lincoln. 


\section{Authors}

S. L. Taylor, S. L. Hefle, C. Bindslev-Jensen, F. M. Atkins, C. Andre, C. Bruijnzeel-Koomen, A. W. Burks, R. K. Bush, M. Ebisawa, P. A. Eigenmann, A. Host, Jonathan O. B. Hourihane, E. Isolauri, D. J. Hill, A. Knulst, G. Lack, H. A. Sampson, D. A. Moneret-Vautrin, F. Rance, P. A. Vadas, J. W. Yunginger, R. S. Zeiger, J. W.

Salminen, C. Madsen, and P. Abbott 


\title{
A consensus protocol for the determination of the threshold doses for allergenic foods: how much is too much?
}

\author{
S. L. Taylor*, S. L. Hefle*, C. Bindslev-Jensen†, F. M. Atkinsł, C. Andre§, C. Bruijnzeel-Koomen $\uparrow$, A. W. Burks\|,

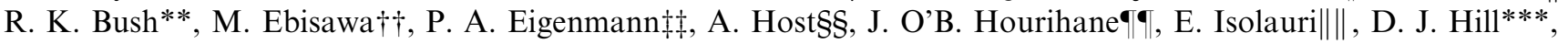

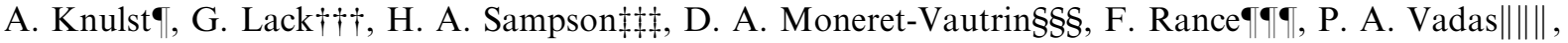

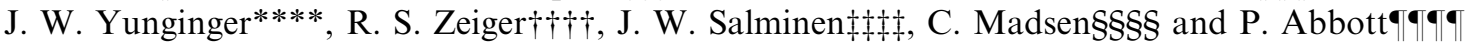 \\ *University of Nebraska, Food Allergy Research and Resource Program, Lincoln, NE, USA, †Department of Dermatology, Odense University Hospital, \\ Odense, Denmark, †National Jewish Medical and Research Center, Denver, CO, USA, §Stallergenes, Antony, France, †Department of Dermatology/ \\ Allergology, University Medical Center, Utrecht, the Netherlands, \|Arkansas Children's Hospital, Little Rock, AR, USA, **William S. Middleton Veterans \\ Administration Hospital, Madison, WI, USA, †National Sagamihara Hospital, Kanagawa, Japan, $\ddagger$ Hopital des Enfants, Geneva, Switzerland, §\$Department \\ of Pediatrics, Odense University Hospital, Odense, Denmark, ФণUniversity of Southampton, Southampton, UK, I||Department of Pediatrics, University of \\ Turku, Turku, Finland, ***Royal Children’s Hospital, Parkville, Australia, ††St Mary’s Hospital, London, UK, $\ddagger \ddagger \ddagger$ Mt Sinai School of Medicine, New York, \\ NY, USA, §§§Department of Internal Medicine, Clinical Immunology and Allergology, University Hospital, Nancy, France, $\uparrow$ Department of Allergologie, \\ Hopital des Enfants, Toulouse, France, |||||Wellesley Central Hospital, Toronto, Ont., Canada, ****Mayo Clinic, Rochester, MN, USA, †††Kaiser \\ Permanente Medical Center, San Diego, CA, USA, łłł Health Products and Foods Branch, Chemical Health Hazard Assessment Division, Ottawa, Canada, \\ $\S \S \S \S$ Danish Veterinary \& Food Administration, Søberg, Denmark and $\uparrow \uparrow \uparrow$ Food Standards Australia New Zealand, Canberra, Australia
}

\begin{abstract}
Summary
Background While the ingestion of small amounts of an offending food can elicit adverse reactions in individuals with IgE-mediated food allergies, little information is known regarding these threshold doses for specific allergenic foods. While low-dose challenge trials have been conducted on an appreciable number of allergic individuals, a variety of different clinical protocols were used making the estimation of the threshold dose very difficult.

Objective A roundtable conference was convened to develop a consensus clinical protocol for lowdose challenge trials for the estimation of threshold doses for specific allergenic foods.

Methods In May 2002, 20 clinical allergists and other interested parties were invited to participate in a roundtable conference to develop consensus of the key elements of a clinical protocol for lowdose challenge trials.

Results A consensus protocol was developed. Patients with convincing histories of food allergies and supporting diagnostic evidence including past challenge trials or high CAP-RAST scores can be enrolled in low-dose challenge trials. Care must be taken with younger patients to assure that they have not outgrown their food allergy. An approach was developed for the medication status of patients entering such trials. Challenge materials must be standardized, for example, partially defatted peanut flour composed of equal amounts of the three major varieties of peanuts (Florunner, Virginia, Spanish). Challenge materials must be appropriately blinded with sensory evaluation used to confirm the adequacy of blinding. A double-blind, placebo-controlled design should be used for low-dose challenge trials. Low-dose challenge trials would begin at doses of $10 \mu \mathrm{g}$ of the allergenic food and would continue with doses of $100 \mu \mathrm{g}$ and $1 \mathrm{mg}$ followed by specific higher doses up to $100 \mathrm{mg}$ depending upon the expert judgement of the physician; even higher doses might be applied to assure that the patient is indeed reactive to the particular food. A 30-min time interval would be used between doses, and reactive doses would be expressed as both discrete and cumulative doses. The goal of each challenge would be to develop objective symptoms; trials should not be discontinued on the basis of subjective symptoms only. Statistically, a minimum of 29 patients would be enrolled in low-dose challenge trials for each allergenic food because 0 reactors out of 29 patients at a particular dose allow the conclusion that there is $95 \%$ certainty that $90 \%$ of allergic individuals will not react to that dose. Conclusion A consensus protocol was developed. Using this protocol, it will be possible to estimate threshold doses for allergenic foods, the lowest amount that elicits mild, objective symptoms in highly sensitive individuals.
\end{abstract}

Keywords allergy, clinical trial, DBPCFC, exposure, food, threshold, risk assessment Submitted 7 July 2003; revised 12 September 2003; accepted 3 November 2003

Sponsored by the Food Allergy Research and Resource Program.

Correspondence: Steve L. Taylor, University of Nebraska, Food Allergy

Research and Resource Program, 143 Food Industry Building, Lincoln,

NE 68583-0919, USA. E-mail: staylor2@unl.edu 


\section{Introduction}

Allergists know that exposure to small quantities of the offending food can elicit adverse reactions in individuals with IgE-mediated food allergies $[1,2]$. While the implementation of strict avoidance diets is prudent clinical advice to give to foodallergic consumers, the assumption of a zero tolerance level for the offending foods places unachievable burdens on the food industry for numerous reasons that have been described previously [3]. Such advice may also unnecessarily restrict the food choices for patients suffering from food allergies. Thus, in 1999, the Food Allergy Research and Resource Programme at the University of Nebraska with sponsorship from the worldwide food industry convened a roundtable conference entitled 'Threshold Doses for Allergenic Foods: How Much is Too Much?' The goal of that initial conference was to determine if data might exist from low-dose, double-blind, placebocontrolled food challenges (DBPCFCs) of individuals with various IgE-mediated food allergies to allow establishment of threshold doses for specific foods.

A threshold dose for allergenic foods is defined for practical purposes as the lowest observed adverse effect level (LOAEL), an amount of a specific food that would elicit mild, objective symptoms in highly sensitive individuals [3]. In the determination of the threshold dose, it would also be desirable to establish the no-observed adverse effect level (NOAEL), an amount of the specific food that elicited no adverse reaction even among individuals with a high degree of sensitivity to that particular food [3]. The establishment of threshold doses and NOAELs for specific allergenic foods may enable regulatory agencies to establish levels for allergenic foods that would protect the vast majority of the sensitive population of consumers. This would provide the food industry with a target to work toward through good manufacturing practices and sanitation programmes. Of course, threshold doses and thus the safe levels of intake are not likely to be the same for all allergenic foods.

Information from DBPCFCs with low doses of the offending food demonstrates rather clearly that finite threshold doses exist below which allergic consumers will not react [3]. Individuals with IgE-mediated food allergies appear to vary rather widely in their degree of sensitivity to specific allergenic foods, for example, in DBPCFCs with peanutallergic individuals, individual threshold doses ranged from 2 to $>50 \mathrm{mg}[4,5]$. Sufficient information existed to indicate that the threshold doses for peanut, egg, and cows' milk were in the low milligram range or higher for individuals with allergies to those specific foods [3]. However, the establishment of NOAELs or LOAELs for specific allergenic foods was not attempted at the 1999 Conference because interpretation of the clinical data was complicated by the use of different clinical challenge protocols, possible biases in the selection of patients for low-dose trials, the failure to record NOAELs during diagnostic challenge trials, and other factors outlined previously [3].

In 1999, the recommendation was made to undertake an international effort to develop standardized clinical challenge protocols for the determination of NOAELs and LOAELs for various commonly allergenic foods [3]. Thus, in May of 2002, the Food Allergy Research and Resource Programme at the University of Nebraska again with sponsorship from the world-wide food industry convened a second roundtable conference on threshold doses for allergenic foods with the goal to develop consensus on the elements of a standardized clinical protocol for low-dose food challenges in highly allergic patients. This manuscript describes the outcome of that second conference.

\section{The consensus clinical protocol}

Threshold doses can only be determined using DBPCFCs with low doses of the offending food. Such trials should be performed in accordance with good clinical practices. Several elements of the challenge protocol should be standardized so that data can be compared from multiple clinical investigators. The key elements are outlined in Table 1. DBPCFCs are already recommended as useful in the diagnosis of food allergies, and many elements of these diagnostic protocols have already been standardized [6, 7]. However, the lowest doses used in diagnostic DBPCFCs have not been standardized, but are instead based upon the nature and severity of the reaction and the lowest amounts thought to provoke adverse reactions from the patient's history [6, 7]. However, typical starting doses for diagnostic DBPCFCs are in the range of $250-500 \mathrm{mg}$ for the most sensitive patients [8,9], well above the levels that are considered in this protocol. However, lower doses are used on occasion with patients suspected to be especially sensitive [3].

Considerable discussion is centred around the need for benefits to accrue to the patients enrolled in such clinical trials in order to obtain ethics board approvals. Individual patients would certainly benefit from learning their individual threshold doses, and that knowledge could benefit them in the implementation of suitable avoidance diets (although they could conceivably react to a lower dose on some future occasion). Certainly, a low-dose challenge trial could also be performed as a component of a normal diagnostic DBPCFC that would be included in the diagnosis of certain patients with certain specific food allergies. A low-dose challenge trial could also be as part of a DBPCFC that is intended to assess whether the patient might have outgrown their allergic sensitization. Such challenges are periodically performed especially on patients where a strong possibility exists that the food allergy may be outgrown (e.g. cows' milk, egg, soybean, wheat) [10]. It may also be possible to couple low-dose challenge trials to trials of novel therapeutic interventions where the determination of threshold doses and the alteration

Table 1. Key elements of the consensus clinical protocol

Selection criteria for patients
Natural history of allergic reactions to specific foods
Medical and pharmacological status of patients immediately prior to challenge
Standardization of challenge materials
Challenge vehicles
Blinding of challenge trial
Dosage range for clinical trial
Time interval between doses
Discrete doses vs. cumulative doses
Interpretation of positive responses: objective vs. subjective symptoms

Natural history of allergic reactions to specific foods

Medical and pharmacological status of patients immediately prior to challenge

Challenge vehicles

Blinding of challenge tria

Dosage range for clinical trial

Discrete doses vs. cumulative doses

Interpretation of positive responses: objective vs. subjective symptoms Standardization of challenge materials 
of the threshold dose in response to the therapy is a necessary part of the trial design. Even in cases where diagnostic criteria obviate the need to perform the DBPCFC as part of the diagnosis, a low-dose challenge trial may be performed to determine whether certain low or very low doses might be tolerated by sensitive individuals.

\section{Patient selection criteria}

The vast majority of patients with convincing histories and documented diagnostic evidence of IgE-mediated allergic reactions to the specific food can be considered for inclusion into low-dose DBPCFCs. Patients with histories of severe anaphylactic reactions may be included in such trials at the discretion of the physician. Although the possible risks to such patients are obvious, no proof exists regarding whether these patients have lower threshold doses than other patients or not. Obviously, inclusion of such patients in low-dose challenge trials would yield valuable information in cases where they are included at the discretion of the physician and with the consent of the patient or patient's family. However, the margin between the threshold dose and the dose that would provoke a severe reaction is not known either; so extreme caution should be applied with such patients.

Patients with evidence of allergic sensitization to the specific food by history together with skin prick test (SPT) or RAST could be included in these trials, although ideally patients enrolled in such trials would have positive DBPCFCs to the food in consideration. For certain foods, CAP-RAST values that provide greater than $95 \%$ probability that the patient will experience an allergic reaction to a specific food have been established $[11,12]$. In some clinics, the CAP-RAST result obviates the need to perform a DBPCFC at the standard doses to establish the diagnosis, although low- or very low-dose challenges may be conducted on such patients including paediatric cases to determine if they are able to tolerate such doses and to aid in the development of suitable avoidance diets. In cases where the CAP-RAST value is below the established level or for foods where no such CAPRAST level has been established, DBPCFCs remain a standard procedure for diagnosis so a low-dose challenge trial could be incorporated a part of this diagnostic procedure. Controversy remains regarding CAP-RAST cut-off values for specific foods [13]. The inclusion of patients with high CAP-RAST values but without positive DBPCFCs in lowdose challenge trials is a matter of expert judgement. Ideally, patients in such trials would have positive DBPCFCs. However, some clinical ethics boards will not allow oral challenges of patients with high CAP-RAST scores, and it may be important to include such patients in low-dose challenge trials.

\section{Natural history of allergic sensitization}

As noted, infants and young children often outgrow their sensitivities to specific allergenic foods, especially cows' milk, eggs, soybeans, and wheat [10]. Obviously, this factor should be borne in mind when selecting patients for enrollment in low-dose challenge trials. With patients where this possibility exists, the DBPCFC should proceed beyond the low doses to higher doses that actually provoke adverse reactions where necessary to assure that a negative response at the lower doses is truly a negative response and not a false-negative reaction associated with loss of allergic sensitization. When such patients have a negative DBPCFC, an open challenge with a typical amount of the particular food is advocated to confirm a loss of allergic sensitization [6].

\section{Medical and pharmacological status of patients prior to challenge}

Certain patients should be excluded from low-dose challenge trials if their allergic conditions are unstable. Examples might include asthma or chronic urticaria. For asthma, the $\mathrm{FEV}_{1}$ should be $80 \%$ of predicted or the patient's best (whichever is greater) immediately before the trial commences (in young children less than 6 years of age, performance of lung function measurements is not possible so in these cases there must be no clinical sign of asthma). Inclusion of patients with highly unstable conditions enhances the likelihood of falsepositive responses. While the exclusion of unstable asthmatics from such trials is prudent, the assumption is that the threshold doses for such patients will not be lower than for other highly sensitive subjects who are enrolled in the trials. Atopic dermatitis and chronic urticaria should also be well controlled before enrolling a patient in a low-dose challenge trial. Other chronic medical conditions may also serve to exclude certain patients from such trials.

Patients enrolled in low-dose challenge trials should be taken off all prescribed medications related to their allergies for suitable withdrawal periods before commencement of the challenge trial. In particular, $\mathrm{H} 1$ antihistamines with a short duration of activity should be withheld for a minimum of $48 \mathrm{~h}$, hydroxyzine needs to be withheld for a minimum of $72 \mathrm{~h}$, leukotriene receptor antagonists should be withheld for up to 1 week, and second generation antihistamines (cetirizine) should be withheld for at least 1 week. In the case of patients with co-existing or food-induced asthma, shortacting inhaled or oral $\beta$-agonists and inhaled anticholinergics could be continued up to $6 \mathrm{~h}$ before challenge. Long-term $\beta$ agonists should be stopped $12 \mathrm{~h}$ before challenge. Chromones (cromolyn and nedocromil) should be withheld for $48 \mathrm{~h}$. Inhaled steroids or oral corticosteroids would be continued in patients where prescribed, although patients with such serious asthma would probably not be included in low-dose challenge studies on most occasions. If patients are taken off of routine medications, the challenge trials must be conducted in a manner that any reactions arising from the withdrawal of medications can be distinguished from true reactions to the food incorporated into the challenge trial. This may require a challenge day composed entirely of placeboes rather than use of interspersed placeboes.

No foods should be ingested for $6 \mathrm{~h}$ prior to challenge except in the case of young children where it would be permitted as necessary. Caffeine and alcohol should also be excluded for $24 \mathrm{~h}$ prior to challenge. While the evidence for caffeine and alcohol withdrawal is limited, alcohol may in some cases exacerbate allergic reactions [14] and caffeine is 
known to have pharmacological effects that could conceivably interfere with observations [15].

\section{Standardization of challenge materials}

The challenge materials should be standardized for use in low-dose challenge trials. One of the biggest confounding variables in existing clinical data on LOAELs is the lack of uniformity in the selection of challenge materials [3]. For example with cows' milk, liquid whole milk, non-fat dry milk, and infant formula have been used as challenge materials in various clinics [3]. Challenge materials should be representative of forms of that food that are commonly used by the food industry and are therefore likely to already have been identified as potential allergen risks to the allergic subjects. However, processing may certainly affect the degree of allergenicity of specific foods even though many commonly allergenic foods are resistant to inactivation during processing [9]. For threshold studies, the form of the particular food used as the challenge material should be one that is known to elicit allergic reactions in most patients with allergies to that specific food. The form of the particular food, barring other considerations, should be one that is particularly well suited to uniform incorporation into various vehicles. Beyond these considerations, some arbitrary agreement is needed simply for the sake of uniformity so that clinical data may be easily compared from various clinics.

Dry materials tend to be more shelf-stable than liquid materials. Thus, non-fat dry milk and spray-dried egg white are advantageous over liquid milk or liquid egg white.

For cows' milk, non-fat dry milk would be the optimal choice in terms of consistency and ease of incorporation into various vehicles. Evidence indicates that processing, including heating (and presumably drying), has no effect on the allergenicity of cows' milk [16].

For egg, spray-dried egg white would be the ideal choice for similar reasons and because most egg-allergic individuals are more sensitive to egg white than to egg yolk [17]. Heat processing, including presumably drying, can lessen the allergenicity of egg [18, 19], but most patients do not experience such differences. Clearly, egg-allergic patients who are sensitive only to raw eggs should be excluded from such trials. Patients sensitive only to raw eggs have simpler avoidance diets and little risk from processed foods.

The choice gets more difficult for peanuts because several different varieties of peanuts are commonly eaten. The group concluded that the ideal challenge material for peanuts should be partially defatted peanut flour composed of equal parts of Florunner, Virginia, and Spanish peanuts; the levels of the major peanut allergens are similar in all varieties of peanuts [20]. Because this type of peanut flour is not commercially available, it would need to be made in a pilot plant facility using conditions similar to those employed in the commercial production of commercial peanut flour. Processing, specifically roasting, enhances the allergenicity of peanuts [21], but peanut-allergic individuals are known to be allergic to peanut flour [4]. Obviously, the threshold dose could differ for roasted peanuts vs. peanut flour but this could be investigated with this type of DBPCFC approach.
For soybean, partially defatted soy flour was selected as the optimal challenge material. Although dozens of soybean varieties are grown commercially, the most common varieties are intermingled commercially and varietal influences on allergenicity are not known to exist. The soybean flour should be free of genetically modified soybeans simply to avoid any possible concern from some ethics boards. Processing may affect the allergenicity of soybeans, especially fermentation and preparation of hydrolysates $[22,23]$. Soybean flour may be the most allergenic form of soybean but the comparative allergenicity of various soybean ingredients requires further study.

Both peanut and soybean flours are typically obtained from heated, rather than raw, seeds and typical commercial practices should be followed in the preparation of these challenge materials. Use of specialized pilot plants to prepare challenge materials (and vehicles) may be necessary in some situations to prepare varietal mixtures, for example, peanuts that thus avoid the necessity of conducting multiple trials on individual varieties, to prevent cross-contamination of raw materials (e.g. grain mixtures that can occur on farms and in distribution and storage), and to provide the best characterized challenge material.

Similar considerations should be employed with other commonly allergenic foods in the selection of the most suitable challenge materials. In some cases such as fish, the selection of appropriate challenge materials may be rather difficult since variations seem to exist in sensitization to specific fish species [24].

\section{Challenge vehicles and blinding of challenges}

The challenge materials should be incorporated into challenge vehicles in a centralized locale and distributed to various clinics participating in the low-dose challenge trial. This approach would maximize consistency and provide the easiest interpretation of the resulting data.

The challenge vehicles should be shelf-stable; refrigeration or freezing causes logistical problems in distribution from a centralized location. Capsules were not recommended because they are difficult to administer to younger patients and they often dissolve slowly in the gastrointestinal tract. Lowfat vehicles should be used since fat slows digestion of protein. The challenge material should be simple to incorporate into the challenge vehicle. An example would be the use of an intensely flavoured gelatin dessert where defined doses of the challenge material might be incorporated into individual small containers. The only steps at the clinic would then be the addition of a defined amount of water, mixing, and refrigeration to allow gelation. Of course, use of gelatin can raise concerns due to religious restrictions and dietary/cultural preferences since it is often derived from pork. Instant oatmeal, instant mashed potatoes, and instant pudding are other possible challenge vehicles that eliminate this concern. Care must be taken to exclude patients with allergies to components of the challenge vehicle, for example, milk in instant pudding.

The challenges should be appropriately blinded. Sensory evaluation should be used with individuals not included in the challenge trial to assure that the challenge material cannot be 
detected in the vehicle at the highest dose. A simple triangle test can be used in sensory evaluation for such purposes [25]. The addition of flavours is important to the ability to mask any flavour associated with the challenge material. Care must be taken to select flavouring materials that do not contain any materials derived from commonly allergenic foods. Flavouring components can occasionally be derived from known allergenic sources [26].

\section{Dosage range for the challenge trials}

For low-dose challenge trials, a double-blind and placebocontrolled design should be employed. Placeboes should be the challenge vehicle alone. Placeboes can be interspersed randomly on a single day where necessary to obtain the patient's consent, but the preferred approach is use of a separate placebo day. As noted previously, a separate placebo day is advisable in situations where the patient has been withdrawn from key medications known to be necessary to control their chronic condition (asthma, urticaria, atopic dermatitis).

Consensus was achieved on an ideal dosage range and challenge protocol to use in these low-dose challenge trials. The consensus was based primarily upon experience obtained with peanuts, cows' milk, and eggs. The dosage range might need to be modified for other allergenic foods based upon expert considerations.

For patients enrolled in these trials on the basis of history, SPT, or CAP-RAST below the $95 \%$ probability value as described in patient selection criteria, the low-dose challenge trial would be incorporated into a normal diagnostic DBPCFC. Doses of $10 \mu \mathrm{g}, 100 \mu \mathrm{g}$, and $1 \mathrm{mg}$ of the allergenic food would be followed by higher doses up to $100 \mathrm{mg}$ depending upon the expert judgement of the physician. A separate placebo day would be used. The order of administration of placebo (first or second trial day) would be random and blinded. A dose of $10 \mu \mathrm{g}$ has never been associated with elicitation of subjective or objective symptoms in any previous low-dose challenge trials so the selection of this dose should be safe even for highly sensitive patients. The inclusion of the $10 \mu \mathrm{g}$ dose also helps to assure that a NOAEL will be determined. The $100 \mu \mathrm{g}$ dose has been implicated in mild, transient, subjective symptoms in one low-dose challenge trial with peanuts [4]. The lowest dose of an allergenic food provoking an objective reaction was $1 \mathrm{mg}$ for peanuts and eggs [3]. If the low-dose challenge is used as a part of a normal diagnostic protocol or as a part of a protocol to determine if a specific allergy has been outgrown, even much higher doses may need to be included to provoke an adverse reaction in most allergic patients.

For patients selected on the basis of a CAP-RAST score that is $>95 \%$ predictive of an allergic reaction, the low-dose challenge trial would be designed to determine doses that the individual patient could tolerate. In this case, the entire trial with randomly interspersed placeboes could be conducted on a single day, but use of a separate placebo day remains the preferred approach. Doses of $10 \mu \mathrm{g}, 100 \mu \mathrm{g}, 500 \mu \mathrm{g}$, and $1 \mathrm{mg}$ of the allergenic food would be used. Based on past experiences with low-dose challenges, a $1 \mathrm{mg}$ dose would be expected to elicit a mild, objective adverse reaction in no more than $5 \%$ of the sensitive patients. This $1 \mathrm{mg}$ dose would be equivalent to 20 p.p.m. in a food with a 50 -g serving size.

\section{Time interval between doses}

The time interval between discrete doses in the low-dose challenge trial should be $30 \mathrm{~min}$. As noted earlier, a $30-\mathrm{min}$ interval does not provide sufficient time for complete assimilation of the challenge material but longer intervals would simply be impractical. With the results presented on the basis of both the cumulative and the discrete doses, any concerns about the selection of the time interval should be abated. The physician could delay the administration of the succeeding dose at their discretion if the situation warranted, for example, the patient complains of subjective symptoms but no objective signs are visible. Provision should also be made for administration of an identical or placebo dose before proceeding to the next higher dose if such a decision is considered prudent by the physician for reasons similar to those noted above.

\section{Discrete vs. cumulative doses}

When positive responses are obtained in low-dose DBPCFCs, the results should be recorded in terms of the discrete dose (the dose administered just prior to the onset of the positive response) and the cumulative dose (the sum of all doses given prior to the onset of the positive response). Using reasonable time intervals including the recommended 30-min intervals, the digestion of discrete doses of the allergenic food and the allergenic proteins contained therein is probably incomplete before administration of the next discrete dose. Therefore, the expression of the results as a cumulative dose is probably justified since a clear break does not occur between individual doses. However, the reporting of both the discrete dose and the cumulative dose is relatively easy and provides the maximum information of later use and interpretation.

\section{Subjective vs. objective responses}

The assessment of patients' responses to the low-dose challenge is clearly a matter of expert clinical judgement. In all cases, the reasons for stopping the challenge sequence should be carefully and thoroughly recorded. Completely subjective symptoms (e.g. mouth itching) should not serve as the basis for cessation of the challenge trial but could serve as a reason to repeat a specific dose in the challenge sequence. Subjective symptoms are potentially meaningful in circumstances where such symptoms are not encountered during the placebo challenge (not known until trial is completed), so subjective reactions should be carefully recorded. However, the progression of the challenge trial to one or more higher doses that elicit an objective symptom is needed before cessation of the trial. Standardized scoring systems should be developed and employed to document any objective symptoms. Observations should be recorded in a uniform manner. For mild objective symptoms (e.g. one or two small urticarial 
lesions that spontaneously resolve), experts might disagree regarding whether the response was a positive response to the challenge. In such situations, a small group of four to five expert allergists should be consulted for their opinions. In these circumstances, photographs should be taken of these symptoms and shared. Again, the development of very mild, objective symptoms could serve as the basis to repeat a specific dose in the challenge sequence.

Many allergic patients have somewhat unpredictable manifestations and normal variability likely exists with respect to mild responses to low-dose challenges. Because reactions at the lowest doses would have profound implications in the identification of LOAELs and NOAELs, the reproducibility of these responses should be sought in certain situations. If mild, objective reactions occur at the very lowest doses in the protocol (either the $10 \mu \mathrm{g}$ or $100 \mu \mathrm{g}$ doses), these responses would be considered especially unusual. In these circumstances, a repeat of the entire low-dose challenge trial should be performed on a different day. If possible, this rechallenge should occur within 7 days with a maximum time interval between challenges of 6 weeks. The minimum time interval between challenges would be $24 \mathrm{~h}$, although a repeat challenge might be possible on the same day if the symptoms resolve spontaneously. If the patient reacts again to the lowest challenge dose $(10 \mu \mathrm{g})$ with objective symptoms, then a NOAEL has clearly not been established; another challenge of this patient with an even lower dose of 1 or $5 \mu \mathrm{g}$ would be desirable in such circumstances.

The interpretation of patient responses to low-dose challenges is an extremely important element of the protocol. The progression of the challenge trial to a point where mild, objective symptoms are encountered serves to remove some potential controversy. Risk assessments should ideally be based on the lowest doses eliciting objective symptoms rather than upon lower doses eliciting subjective symptoms. Even in circumstances where a patient experiences subjective symptoms at a low dose that are confirmed by objective symptoms at higher doses, the risk assessment should be based upon the doses eliciting objective symptoms. In low-dose challenge trials with peanuts, subjective symptoms were encountered in some patients at doses $(100 \mu \mathrm{g})$ that were 20 -fold lower than the lowest doses provoking mild objective reactions [4]. As noted previously, the threshold dose is defined as the LOAEL that elicits mild, objective symptoms in highly sensitive individuals [3]. In cases where repeat challenges are performed that yield differing results at the same low doses on separate occasions, the positive result cannot be discounted in the risk assessment. However, the variability in the response could be taken into account.

\section{Statistical interpretation and risk assessment}

The goal to be achieved from standardized and comparable low-dose challenges of sensitive patients would be the establishment of threshold levels that would protect the vast majority of allergic consumers. A typical approach would be to conduct low-dose challenges using this standardized protocol on a total of 29 patients with a specific food allergy. If a level can be identified that does not elicit an adverse reaction in any of the 29 subjects, then statistically it can be concluded that $95 \%$ certainty exists that $90 \%$ of allergic individuals will not react to this dose of the particular allergen. While this level of assurance clearly does not protect all patients, this approach has been advocated in the development of hypoallergenic infant formula [27]. While hypoallergenic infant formula is safe for the vast majority of cows' milk-allergic infants, occasional allergic reactions are noted in exquisitely allergic infants [28-32]. The only method to determine if a particular threshold dose would protect a specific patient would be to conduct a low-dose DBPCFC.

Alternatively, existing clinical data from low-dose challenges from multiple clinics using somewhat different protocols could be assessed statistically. This approach was recently tried by Bindslev-Jensen et al. [33] using published data from the clinical literature. However, this approach is complicated by the uncertainties associated with failure to identify a NOAEL in most existing observations, the effects of differences in the protocols, and other factors [33]. Using this approach, threshold values that would protect 99 of 100 allergic individuals were $8.6 \mathrm{mg}$ (milk), $3.4 \mathrm{mg}$ (egg), $1.2 \mathrm{mg}$ (peanut), and $2.2 \mathrm{mg}$ (soybean) [33]. The reliability of these estimates would improve with data from standardized clinical protocols.

Additionally, the risk assessment will likely need to include expert clinical and regulatory judgement beyond simple statistical interpretations. Other factors such as the seriousness of the reaction, objective vs. subjective reactions, and the inclusion of uncertainty factors to account for the possible existence of more highly sensitive individuals will also need to be considered. Also, the expression of the threshold dose will need to include an acceptable degree of risk. Bindslev-Jensen et al. [33] estimated threshold doses for degrees of risk ranging from one in a million to one in a hundred. No international agreement has been reached on an acceptable level of risk for allergic individuals. Thus, further discussion regarding the application of risk assessment principles to actual data from low-dose challenges will be anticipated once data become available from the standardized clinical protocol outlined here.

\section{References}

1 Laoprasert N, Wallen ND, Jones RT, Hefle SL, Taylor SL, Yunginger JW. Anaphylaxis in a milk-allergic child following ingestion of lemon sorbet containing trace quantities of milk. J Food Prot 1998; 61:1522-4.

2 Gern JE, Yang E, Evrard HM, Sampson HA. Allergic reactions to milk-contaminated 'non-dairy' products. N Engl J Med 1991; 324:976-9.

3 Taylor SL, Hefle SL, Bindslev-Jensen C et al. Factors affecting the determination of threshold doses for allergenic foods: how much is too much. J Allergy Clin Immunol 2002; 109:24-30.

4 Hourihane JO'B, Kilburn SA, Nordlee JA, Hefle SL, Taylor SL, Warner JO. An evaluation of the sensitivity of subjects with peanut allergy to very low doses of peanut: a randomized, double-blind, placebo-controlled food challenge study. J Allergy Clin Immunol 1997; 100:596-600.

5 Wensing M, Penninks AH, Hefle SL, Koppelman SJ, BruijnzeelKoomen CAFM, Knulst AC. The distribution of individual threshold doses eliciting allergic reactions in a population with peanut allergy. J Allergy Clin Immunol 2002; 110:915-20. 
6 Bock SA, Sampson HA, Atkins FM et al. Double-blind, placebocontrolled food challenge (DBPCFC) as an office procedure: a manual. J Allergy Clin Immunol 1988; 82:986-97.

7 Bindslev-Jensen C. Standardization of double-blind, placebocontrolled food challenges. Allergy 2001; 56 (Suppl. 67):75-7.

8 Sampson HA. Role of immediate hypersensitivity in the pathogenesis of atopic dermatitis. J Allergy Clin Immunol 1983; 71:473-80.

9 Taylor SL, Lehrer SB. Principles and characteristics of food allergens. Crit Rev Food Sci Nutr Suppl 1996; 36:91s-118s.

10 Bock SA. The natural history of food sensitivity. J Allergy Clin Immunol 1982; 69:173-7.

11 Sampson HA, Ho D. Relationship between food-specific IgE concentration and the risk of positive food challenges in children and adolescents. J Allergy Clin Immunol 1997; 100:444-51.

12 Sampson HA. Utility of food-specific IgE concentrations in predicting symptomatic food allergy. J Allergy Clin Immunol 2001; 107:891-6.

13 Osterballe M, Bindslev-Jensen C. Threshold levels in food challenge and specific IgE in patients with egg allergy: is there a relationship? J Allergy Clin Immunol 2003; 112:196-201.

14 Betschel SD, Vadas P. Ethanol is a requisite cofactor in anaphylaxis to spinach. J Allergy Clin Immunol 2003; 111:S100.

15 Maher TJ. Pharmacological actions of food and drink. In: Brostoff J, Challacombe SJ, eds. Food allergy and intolerance, 2nd Edn. London: WB Saunders, 2002; 445-51.

16 Host A, Samuelsson EG. Allergic reactions to raw, pasteurized, and homogenized/pasteurized cow milk: a comparison. Allergy 1988; 43:113-8.

17 Anet J, Back JF, Baker RS, Barnett D, Burley RW, Howden $\mathrm{MEH}$. Allergens in white and yolk of hen's egg: a study of IgE binding by egg proteins. Int Arch Allergy Appl Immunol 1985; 77:363-71.

18 Eigenmann PA. Anaphylactic reactions to raw eggs after negative challenges with cooked egg. J Allergy Clin Immunol 2000; 105:587-8.

19 Romeira AM, Pires G, Gaspar A, Arede C, Morais-Almeida M, Rosado-Pinto J. Egg allergy - to be or not to be boiled. Allergy 2003; 58:533-4.

20 Koppelman SJ, Vlooswijk RAA, Knippels LMJ et al. Quantification of major peanut allergens Ara h 1 and Ara h 2 in the peanut varieties Runner, Spanish, Virginia, and Valencia, bred in different parts of the world. Allergy 2001; 56:132-7.

21 Maleki SJ, Viquez O, Jacks T et al. The major peanut allergen, Ara h 2, functions as a trypsin inhibitor, and roasting enhances this function. J Allergy Clin Immunol 2003; 112:190-5.

22 Herian AM, Taylor SL, Bush RK. Identification of soybean allergens by immunoblotting with sera from soy-allergic adults. Int Arch Allergy Appl Immunol 1990; 92:193-8.

23 Burks AW, Williams LW, Thresher W, Connaughton C, Cockrell G, Helm RM. Allergenicity of peanut and soybean extracts altered by chemical or thermal denaturation in patients with atopic dermatitis and positive food challenges. J Allergy Clin Immunol 1992; 90:889-97.

24 Bernhisel-Broadbent J, Scanlon SM, Sampson HA. Fish hypersensitivity. I. In vitro and oral challenge results in fish-allergic patients. J Allergy Clin Immunol 1992; 89:730-7.

25 Stone H, Sidel J. Sensory evaluation practices, 2nd Edn.. Orlando, FL: Academic Press, 1992.

26 Taylor SL, Dormedy ES. The role of flavoring substances in food allergy and intolerance. Adv Food Nutr Res 1998; 44:1-44.

27 Subcommittee on Nutrition and Allergic Disease. Infant formulas and allergic disease. Report of American Academy of Pediatrics, 1990:47pp.

28 Saylor JD, Bahna SL. Anaphylaxis to casein hydrolysate formula. J Pediatr 1991; 118:71-4.

29 Rosenthal E, Schlesinger Y, Birnbaum, Goldstein R, Benderly A, Freier S. Intolerance to casein hydrolysate formula. Acta Paediatr Scand 1991; 80:958-60.

30 De Boissieu D, Matarazzo P, Dupont C. Allergy to extensively hydrolyzed cow milk proteins in infants: identification and treatment with an amino acid-based formula. J Pediatr 1997; 131:744-7.

31 Lifshitz CH, Hawkins HK, Guerra C, Byrd N. Anaphylactic shock due to cow's milk protein hypersensitivity in a breast-fed infant. J Pediatr Gastroenterol Nutr 1988; 7:141-4.

32 Nilsson C, Oman H, Hallden G, Lilja G, Lundberg M, Harfast B. A case of allergy to cow's milk hydrolysate. Allergy 1999; 54:1322-6.

33 Bindslev-Jensen C, Briggs D, Osterballe M. Can we determine a threshold level for allergenic foods by statistical analysis of published data in the literature? Allergy 2002; 57:741-6. 